\title{
LA REFLEXIÓN CRÍTICA SOBRE LOS NEUROMITOS EN LA EDUCACIÓN
}

\section{The Critical Reflection of Neuromyths in Education}

\author{
Daniel PALLARÉS-DOMÍNGUEZ
}

Universitat Jaume I de Castellón. España.

Instituto de Enseñanza Secundaria Francesc Ribalta de Castellón. España. dpallare@uji.es; danipado.neuro@gmail.com

bttps://orcid.org/0000-0002-6510-8448

Fecha de recepción:15/12/2020

Fecha de aceptación: 16/03/2021

Fecha de publicación en línea: 01/07/2021

Cómo citar este artículo: Pallarés-Domínguez, D. (2021). La reflexión crítica sobre los neuromitos en la educación. Teoría de la Educación. Revista Interuniversitaria, 33(2), 87-106. https://doi.org/10.14201/teri.25288

\section{RESUMEN}

El progreso de la neuroeducación en los últimos años ha tenido incidencia en la comprensión actual de la educación. Como ciencia interdisciplinar, la neuroeducación ha aportado grandes ventajas sobre la descripción de los correlatos neurales en el proceso de enseñanza-aprendizaje, así como en la detección de los neuromitos. Sin embargo, sus aportaciones no están carentes de una revisión crítica desde la perspectiva filosófica que vaya más allá del cuestionamiento puramente neurocientífico sobre la validez de las evidencias. El objetivo de este artículo es señalar la necesaria aceptación crítica de los resultados de la neuroeducación para evitar un colonialismo neurocientífico como un reto al que la teoría de la educación debe enfrentarse.

Para ello, en primer lugar se justificará la introducción de la neuroeducación en el panorama educativo actual. En segundo lugar, se conceptualizará brevemente la neuroeducación y los neuromitos para poder después, a través de una metodología 
argumentativa y crítica, incidir en los factores de la aceptación de sus descubrimientos. En tercer lugar, para ejemplificar la aceptación crítica de estos, se analizarán las Inteligencias Múltiples de H. Gardner y su posible consideración como neuromito. Este análisis permitirá observar como no siempre las evidencias neurocientíficas, o falta de estas, son suficientes para considerar una teoría educativa peyorativamente como un neuromito. La discusión radica en la consideración de una falta de evidencia científica desde el diálogo interdisciplinar de la neuroeducación. A pesar del abierto debate sobre su consideración neurocientífica y sobre su utilidad a nivel pedagógico, las Inteligencias Múltiples están en sintonía con las consideraciones de las leyes educativas en los últimos años, las cuales han incidido de manera significativa en las competencias de aprendizaje, y en las metodologías y evaluaciones que comprendan al ser humano desde un punto de vista holístico, abriendo así la puerta al diálogo con otras ciencias.

Palabras clave: neuroeducación; neuromitos; Inteligencias Múltiples; interdisciplinariedad; crítica.

\section{ABSTRACT}

The progress of neuroeducation in recent years has had an impact on the current understanding of education. As an interdisciplinary science, neuroeducation has provided great advantages over the description of neural correlates in the teaching-learning process, as well as in the detection of neuromyths. However its contributions are not lacking in a critical review from a philosophical perspective that goes beyond the purely neuroscientific questioning of the validity of the evidence. The main objective of this article is to point out the necessary critical acceptance of the neuroeducation results in order to avoid a neuroscientific colonialism as a challenge that educational theory must face up to.

Firstly we will justify the introduction of neuroeducation in the current educational landscape. Secondly we will conceptualize neuroeducation and neuromyths in order to later on, through an argumentative and critical methodology, analyze the factors behind the acceptance of its discoveries. Thirdly, the Multiple Intelligences theory of H. Gardner will be analyzed in order to exemplify these factors. This analysis will allow us to observe how neuroscientific evidence or lack of them is not always sufficient to consider an educational theory pejoratively as a neuromyth. The discussion lies in the consideration of a lack of scientific evidence from the interdisciplinary dialogue of neuroeducation. Despite of the open debate on its neuroscientific and on its usefulness at the pedagogical level, Multiple Intelligences theory are in tune with the considerations of educational laws in recent years, which have had a significant impact on learning competencies, and on methodologies and evaluations that understand the human being from a holistic point of view, thus opening the door to dialogue with other sciences.

Key words: neuroeducation; neuromyths; multiple intelligences; interdisciplinarity; critical. 


\section{INTRODUCCIÓN}

Han pasado casi dos décadas desde el nacimiento de lo que se ha dado en llamar las neurociencias sociales, entendidas como la aplicación de procedimientos del ámbito neurocientífico para complementar el estudio de las ciencias humanas y sociales (Adolphs, 2010). Lo que comenzó como potenciales aplicaciones de cómo razonamos y sentimos en referencia a operaciones económicas, decisiones políticas, patrones de compra, dilemas morales o procedimientos de aprendizaje, se ha fraguado en forma de disciplinas con un campo de estudio propio, tales como la neuroeconomía, la neuropolítica, el neuromarketing, la neuroética y la neuroeducación.

Esta última, la neuroeducación, se ha ido introduciendo poco a poco en el proceso de enseñanza-aprendizaje especialmente desde varias perspectivas. Es decir, no solo mostrando cómo aprendemos, sino revisando otros presupuestos básicos que se daban por válidos durante dicho proceso y que ahora, debido al mayor conocimiento del cerebro humano, se pueden cuestionar por las evidencias empíricas con las que se cuentan. Se entiende por neuromitos los errores de interpretación generados por una mala comprensión, lectura o cita de evidencias neurocientíficas y aplicadas al contexto educativo (OECD, 2002). Unas evidencias que, en muchos casos, no están exentas de una evaluación crítica desde el punto de vista más humano y filosófico de la educación.

El objetivo central de este artículo es evaluar de qué forma se están aceptando o rechazando los neuromitos en la educación. Para ello, y desde una metodología crítica y argumentativa, es necesario recordar en primer lugar, el cambio de orientación educativa que se ha dado en las últimas décadas, y que ha abierto la puerta a la entrada de las neurociencias en la educación. En segundo lugar, se justificará que la aceptación de los descubrimientos neurocientíficos en el ámbito de la educación debe hacerse bajo un horizonte de sentido crítico y humano, evitando así un colonialismo neurocientífico que, en ocasiones también se debe a una mala traducción de la psicología de la educación. En tercer lugar, y para ejemplificar esta relación, se tomará el caso concreto de un supuesto neuromito, el de las Inteligencias Múltiples de H. Gardner, para reflexionar sobre si las aportaciones neurocientíficas son siempre válidas o no desde el punto de vista educativo para justificar la aceptación o rechazo de teorías que, como en este caso, marcan tan profundamente la práctica educativa.

\section{CONCEPTUALIZACIÓN DE LA NEUROEDUCACIÓN}

\subsection{La posibilidad de conjunción entre las neurociencias y la educación}

Según A. Campos (2010, p. 3), hace poco más de dos décadas que las neurociencias han posibilitado una mayor comprensión acerca del cerebro en el contexto educativo. Este conocimiento mayor sobre el cerebro humano, de forma interdisciplinar 
con otras ciencias, especialmente la psicología de la educación, ha tenido incidencia en el proceso de enseñanza-aprendizaje. El hecho de que ya no se hable solamente de práctica educativa, sino de proceso de enseñanza-aprendizaje, implica tener en cuenta varias capacidades ya no solo de tipo cognitivo, sino también social, emocional, moral y físico sobre los discentes. Por tanto, ¿qué ha abierto la puerta a las neurociencias en el campo educativo? Lejos de realizar una descripción de la evolución de la neuroeducación hasta nuestros días que ya se apuntó en otros trabajos (Pallarés-Domínguez, 2015, 2016), la respuesta no solo se debe a los avances en las propias neurociencias sociales, las cuales tienen mucho que decir, sino también en la educación, quien ha estado dispuesta a escuchar y dialogar. Es decir, la posibilidad de que las neurociencias entren en la educación se ha dado en gran parte por el cambio del horizonte de sentido de la educación en las últimas décadas. Un cambio que pasa por la reconceptualización de la propia escuela en sí — hacia una escuela inclusiva- y el modelo general de enseñanza — de los contenidos a las competencias.

John Dewey definió la educación como «una constante reorganización o reconstrucción de la experiencia que da sentido a la que ya se tiene y aumenta la capacidad para dirigir el curso de la subsiguiente» (Dewey, 1995, p. 91). El núcleo fundamental de esta definición radica en el reconocimiento de toda experiencia vital como fuerza de movimiento y de cambio. Este movimiento experiencial tiene dos direcciones fundamentales que remiten a la doble raíz etimológica de la palabra «educación». En primer lugar, educare, que significa alimentar, criar, nutrir, e incluso conducir, y que se refiere al movimiento de la experiencia "desde fuera hacia dentro» del discente. En segundo lugar, educere, que significa, sacar, extraer, dar a luz, hacer salir o extraer de dentro a fuera, y se refiere al movimiento de la experiencia que sale del discente hacia fuera, a su potencial y al aprovechamiento de sus capacidades

Sin embargo, parece ser que el marco general de la actividad educativa ha tendido a considerar solamente una sola de las raíces del término, la de educare, y por tanto solamente una dirección en el movimiento de la educación, la que va desde el/la docente hacia el alumnado. Afortunadamente en los últimos años, tanto las metodologías, como los tipos de evaluación y las orientaciones del currículo, han hecho eco de una demanda de cambio. Un cambio que vaya desde un paradigma educativo basado en modelos algorítmicos, fijos, y orientados a los contenidos - paradigma tradicional de la educación - a un paradigma educativo basado en modelos heurísticos, y orientado a competencias. Este cambio es evidencia de una necesidad que va más allá del contexto formal de la educación, para abarcar una perspectiva antropológica a la vez que vital. Este cambio pasa por renovar el entorno socioeducativo que perpetuaba un modelo de pensamiento homogéneo, amparado en un paradigma racional instrumental, en un sujeto neoliberal, y en 
una concepción de la educación como progreso de la ciencia y la técnica desde la concepción postmoderna de razón (Formosinho et al., 2013).

Esta concepción se basaba en la mercantilización de los contenidos y en el reduccionismo del ser humano a ellos, evaluando «objetivamente» —según el criterio cientificista moderno- solamente aquello que el mercado demanda, y que casi siempre son saberes técnicos en detrimento de las humanidades, y más aún, interesándose solo en los estudiantes "excelentes» y rehuyendo a los más necesitados (Rogero, 2015). A su vez, ese corporativismo, individualismo y sometimiento de la educación a las leyes del mercado es lo que ha producido y reproducido la cultura del "fracaso escolar» como si se hablara en el contexto de una empresa (Escudero, 2009).

Sin embargo, no debemos olvidar que lo más útil para el ser humano no es una cuestión meramente técnica sino también humana. El ser humano nunca actuará como una "persona total» si dejamos que la razón instrumental y el tecnicismo se adueñen de nuestras vidas (Bauman, 1993). En efecto, ¿debería ser la educación una herramienta al servicio de los poderes económicos y centrada profesionalización técnica que exige el mundo laboral? ¿O debería ser una formación integral, holística y cívica de las personas que contribuya a la forja de valores éticos para una vida en sociedad?

En Europa hemos asistido en las últimas dos décadas a un cambio radical en la enseñanza, desde los contenidos hacia las competencias, los valores están presentes en el mismo concepto central de competencia. Según el Ministerio de Educación, Cultura y Deporte de España, no es posible llevar a cabo una educación adecuada si no es conociendo la transversalidad de los valores éticos. Por otro lado, atendiendo a las Recomendaciones 2006/962/EC del Parlamento Europeo, se insiste en que una buena educación en actitudes y valores éticos es fundamental no solo para la búsqueda de empleo sino también para una ciudadanía activa y una buena inclusión social. La Orden ECD 65/2015, por la que se describen las relaciones entre las competencias, los contenidos y los criterios de evaluación de la educación primaria, la educación secundaria obligatoria y el bachillerato, contempla explícitamente la "competencia social y cívica" como básica en la educación, dado que se basa en «el conocimiento crítico de los conceptos de democracia, justicia, igualdad, ciudadanía y derechos humanos y civiles» (MEC, 2015, p. 13).

La nueva ley educativa sigue apostando por esta línea. Al margen de la discusión política, el proyecto de ley orgánica presentado recientemente tiene como ejes principales la inclusión, la equidad, la sostenibilidad, la mejora del nivel competencial, la educación en el respeto a los derechos y libertades fundamentales, la formación para la paz, el respeto a los derechos humanos, la vida en común y la cohesión social, así como la preparación para el ejercicio de la ciudadanía (Boletín Oficial del Estado, 2020). 
Precisamente es este componente ético, reflexivo y orientado hacia una educación más humana, es lo que ha impulsado en gran medida la reacción frente al paradigma anterior, y ha posibilitado demandas de cambio desde la propia educación hacia perspectivas más críticas y dialógicas, o comunitarias y cooperativas, desde la investigación-acción, o desde las neurociencias, como factores de innovación educativa.

\subsection{El auge de la neuroeducación}

En el apartado anterior se ha justificado cómo el cambio de dirección educativa en los últimos años ha posibilitado la entrada de las neurociencias en la educación. El hecho de apostar no solo por un modelo orientado a las competencias, sino que tiene en cuenta de una forma holística el proceso de enseñanza-aprendizaje del ser humano teniendo como horizonte de sentido su educación en valores éticos y humanos, así como emocionales (Darder \& Bach, 2006), favorece la colaboración interdisciplinar con otras ciencias que nos ayuden a comprender mejor cómo aprendemos. En este sentido, la neuroeducación ha sido una de ellas, especialmente por su perspectiva descriptiva de cómo aprende el cerebro. Sin embargo, la razón instrumental y técnica que se ha descrito en el apartado anterior, no solo afectaba a la educación en algunos aspectos, sino que también ha impregnado una parte de las neurociencias. Por lo que, en la conjunción de las neurociencias con la educación, no se debe de perder de vista el horizonte humano, aceptando de una forma crítica los resultados, en apariencia, tan prometedores de las neurociencias.

La progresiva introducción de los estudios neurocientíficos en las metodologías y procesos de enseñanza aprendizaje se conoce con el nombre de neuroeducación (Goswami, 2006, 2010). Fue una de las últimas ciencias sociales en emerger tras la década del cerebro en 1990, y a partir del año 2002 empezó a adquirir especial relevancia con la publicación del informe de la OECD Understanding the Brain: Towards the New Learning Science.

Para algunos autores, la neuroeducación es una neurociencia cognitiva aplicada más (Ansari, 2005; Ansari \& Coch, 2006; Ansari et al., 2011; Campbell, 2011), por lo que el diálogo con la educación no afectaría a sus descubrimientos ni sería tan necesario para una aplicación directa de los descubrimientos. En cambio, para otros como A. Battro y D. Cardinali (1996), la neuroeducación es, desde una perspectiva integradora y pedagógica, una nueva interdisciplina que promueve una mayor integración de las ciencias de la educación con aquellas que se ocupan del desarrollo neurocognitivo de la persona. También H. Koizumi la definición en esta línea, entendiendo la neuroeducación como una transdisciplina de los procesos de desarrollo de aprendizaje humano (Koizumi, 2008). En este caso, tanto neurocientíficos como educadores intercambiarían puntos de vista para optimizar sus respectivas contribuciones a un proyecto común. 
Sin embargo, hablar de interdisciplinariedad en general es bastante ambiguo, pues lo que define esta disciplina es la forma que adquiere esa relación entre las disciplinas que la forman. Es decir, la relación entre neurociencia y educación podría conducirnos tanto a que la agenda de la neurociencia quede determinada por las necesidades educativas - la investigación en torno a las dificultades de aprendizaje-, como a la aplicación directa de los resultados neurocientíficos en el aula (Howard-Jones, 2011, pp. 119-123). Por tanto, dentro de este abanico de posibilidades es necesaria una cierta prudencia filosófica.

Aun así, el quehacer de esta disciplina ha ido adquiriendo su forma a partir de una bidireccionalidad dialógica entre la neurociencia y la educación, en la que se pueden diferenciar al menos cuatro niveles de relación (Marina, 2012; PallarésDomínguez, 2019). Un primer nivel descriptivo en el que las técnicas neurocientíficas, especialmente las de neuroimagen, nos ayudarían a entender las bases fisiológicas sobre cómo aprende el cerebro. Un segundo nivel de revisión de los presupuestos que las metodologías han asumido como correctas en el proceso de enseñanzaaprendizaje. Un tercer nivel terapéutico o clínico que se dedicaría a investigar los correlatos neurales de las dificultades de aprendizaje. Y por último, un nivel prescriptivo que, aun estando todavía lejano, intentaría crear metodologías de aprendizaje más eficientes teniendo en cuenta cómo funciona el cerebro.

No han faltado las críticas a la imposibilidad de tal proyecto, como las de J. Bruer (1997), quien señaló tres puentes lejanos en el terreno neuroeducativo. En primero se refería al poco conocimiento de la estructura funcional del cerebro para poder unirlo a la práctica educativa. El segundo se refería a la brecha entre la educación y la psicología cognitiva. El tercero a la distancia que existía entre la psicología cognitiva y la neurociencia. A pesar de estas críticas que el propio autor suavizó con los años (Bruer, 2005), y el acercamiento de estos puentes que han producido los dos grandes megaproyectos internacionales - The Human Brain Project, y el Brain Activity Map (Kandel et al., 2013)—, siguen existiendo ciertos problemas de relación por ahora insalvables. Especialmente en el marco conceptual entre la educación y la neurociencia.

Conceptos como inteligencia, aprendizaje, memoria, o mente todavía están lejos de confluir en ambas disciplinas (Davis, 2004, pp. 23-27; Willingham, 2009, p. 545). Pero también en las condiciones en que se realizan los experimentos, y no solo por los riesgos éticos que plantean (Howard-Jones, 2011, pp. 202-212), sino por las condiciones y ambientes controlados de los mismos, muy lejos de la realidad compleja y multidimensional del ambiente real del aula (Schumacher, 2007, p. 43). Por no mencionar que los procesos de enseñanza-aprendizaje son en su mayoría forjados a través de varias personas, como mínimo un docente y un discente, mientras que los experimentos se llevan a cabo en su mayoría solamente en un solo sujeto.

Aún así, el avance de la neuroeducación no se ha detenido por estas dificultades, y se ha centrado en los últimos años especialmente en dos ámbitos de conocimiento, 
el lenguaje y las matemáticas. En parte también por los trastornos del aprendizaje más notables que plantean dificultades en estas asignaturas, como la dislexia o la discalculia. Pero también el nivel de revisión ha ocupado gran parte de su agenda, concretamente en el descubrimiento de los neuromitos.

\section{NEUROMitos Y SU ACEPTACIÓN CRÍtiCA}

\subsection{El tratamiento de los neuromitos en la educación}

A pesar de las dificultades señaladas en el apartado anterior, la función de revisión que aporta la neuroeducación ha revelado que algunos de los supuestos en los que se basaban las metodologías de aprendizaje no coincidían con la forma en que funciona el cerebro. Estas malinterpretaciones que se conocen con el nombre de neuromitos. En este apartado, más que presentar con detalle todos ellos, se abordará de qué forma se aceptan o se rechazan en la educación una vez han sido identificados, así como las justificaciones que, desde el punto de vista filosófico, podríamos tener para su aceptación crítica.

Originariamente, el término neuromito proviene del campo de la medicina, donde en la década de 1980 lo utilizó A. Crockard para describir una falta de relación entre el conocimiento científico sobre el cerebro y las evidencias científicas (Howard-Jones, 2014). Trasladado al ámbito educativo, a partir de 2002 por la OECD, se entienden como creencias generalizadas, debido a extrapolaciones o relaciones apresuradas, que introducen una falsa relación causal entre una metodología educativa y su correspondiente demostración neurocientífica (OECD, 2002; Geake, 2008). Estas malinterpretaciones o falsas creencias son muy difíciles de erradicar porque se expanden con facilidad al tratarse de explicaciones aparentemente simples que han trascendido las condiciones de un laboratorio sin ser debidamente contrastadas, y/o han sido mal comunicadas por los medios de información (Pasquinelli, 2012).

Desde su primera aparición, son comunes los trabajos acerca de la prevalencia de los neuromitos en el ámbito educativo, realizados a través de encuestas de carácter sociológico a los educadores para evaluar los conocimientos que tienen sobre el cerebro, actualizándose con relativa rapidez tanto en el ámbito internacional (Herculano-Houzel, 2002; Gleichgerrtch et al., 2015; Dündar \& Gündüz, 2016; Hermida et al., 2016; Kim \& Sankey, 2017; Zhang et al., 2019) como en el nacional (Fuentes \& Risso, 2015; Ferrero et al., 2016; Medel Montero \& Camacho Conde, 2019).

Entre los neuromitos más comunes se pueden encontrar: los seres humanos utilizamos el 10\% de nuestro cerebro; el condicionamiento del aprendizaje hasta los tres años; la utilización preferente de un hemisferio como condicionamiento del aprendizaje; los estilos preferentes de aprendizaje en base a modalidades sensoriales - visual, auditivo o kinestesico; el hecho de estar extra hidratado es un factor determinante para el aprendizaje; los efectos beneficiosos de ciertos suplementos 
alimenticios en el éxito académico, entre otros. Algunas de las revisiones sistemáticas más actuales llegan a catalogar hasta cuarenta diferentes (Torrijos-Muelas et al., 2020).

A pesar de la gran proliferación de trabajos sobre los neuromitos en educación, y su cuestionamiento desde el punto de vista neurocientífico, también han crecido las investigaciones de carácter crítico sobre la aceptación de los neuromitos y la credibilidad que se les otorga desde el punto de vista filosófico, sociológico y pedagógico (Wolfe, 2006; Bowers, 2016). Si la neuroeducación es una ciencia interdisciplinar, ¿qué sentido tiene que las decisiones puramente neurocientíficas sean las que determinen el auge o declive de una metodología concreta? En el caso de algunas creencias populares que no trascienden al plano pedagógico, estaría más que justificado que la última palabra la tenga la neurociencia. Pero ¿qué ocurre en el caso de otras teorías que pueden tener una incidencia directa en las metodologías de aprendizaje, y que han demostrado ser útiles desde el punto de vista educativo?

La aceptación crítica de estos neuromitos implicaría cuestionarse, al menos, ¿cómo son las evidencias a las que se hace referencia para justificar su aceptación como neuromitos y consecuentemente su rechazo en el proceso de enseñanzaaprendizaje?

En cuanto a las evidencias neurocientíficas (a), se debería cuestionar qué se observa realmente con las técnicas de neuroimagen - entre otras-y por qué adquieren una pertinencia normativa tan grande.

La pretensión de las ciencias naturales para explicar el conjunto de las ciencias sociales y humanas desde supuestos causales no es algo nuevo. El positivismo científico siempre ha tenido esta pretensión. La razón instrumental que denunciaron los filósofos de la primera generación de la Escuela de Franckfurt, o el reduccionismo epistemológico que resaltaron los de la segunda, conlleva la eliminación de cualquier aportación que realicen las ciencias que no funcionen bajo los mismos métodos de observación y verificación empírica de hipótesis que las ciencias naturales (Habermas, 2006, p. 172).

Ese mismo objetivo reduccionista y naturalista sigue estando vigente, ahora bajo el prefijo "neuro", revestido de un aura de objetividad que mediante la visión de una neuroimagen - y de otras evidencias - y que da la impresión de no necesitar ninguna interpretación humana al respecto. De esta forma, y sin un cuestionamiento crítico de lo que se observa, se corre el peligro de caer en lo que algunos han llamado "neuropositivismo» o "neuropoder», refiriéndose a un claro colonialismo científico y las consecuencias que de éste se derivan (García-Marzá, 2016, p. 886; 2012, p. 83).

A pesar del gran atractivo que puedan tener las supuestas observaciones de la actividad cerebral, ¿debería dárseles tanto crédito? Algunos autores han investigado la seducción que ejercen las evidencias neurocientíficas en el público, concluyendo que generalmente se da mayor valor científico a aquellos estudios que van acompañados de neuroimágenes (Weisberg et al., 2008, p. 475-476; McCabe \& Castel, 2008). Incluso serían más poderosas cuando van acompañadas de una explicación 
del cerebro en términos naturalistas y reduccionistas (Gruber \& Dickerson, 2012). Aún así, esta creciente pertinencia normativa no sería posible sin el creciente interés del público en general por la neurociencia, cambiando por completo los canales de comunicación científica y haciéndola más accesible a todos, y permitiendo su entrada en el debate público, institucional y político (Iles et al., 2010; Racine, 2011, p. 787).

Desde la filosofía, especialmente desde las éticas aplicadas, ya se ha advertido de las confusiones comunes en las que caen quienes otorgan tal poder a las evidencias neurocientíficas. Las falacias ocupan un lugar común entre éstas. Este reduccionismo de las neurociencias sociales suele tener predilección por la falacia naturalista (Gracia, 2018, p. 210-212), por la confusión entre la razón necesaria y la razón suficiente, y por la confusión entre las bases y los fundamentos (Cortina, 2011, p. 46). Es decir, la observación empírica de un suceso aislado del cerebro - pongamos por caso la mayor concentración de deoxihemoglobina en una parte concreta de éste- quizá suponga la base natural y una razón necesaria para explicar un fenómeno. Pero no por ello constituye la razón suficiente y el fundamento educativo de lo que se pretenda explicar. En el contexto que nos ocupa, la educación es una ciencia fundamentalmente humana, y su carácter interdisciplinar requeriría de una mejor comunicación entre científicos y educadores (Ansari, et al., 2012). Dejar la totalidad última de las explicaciones sobre su proceder a la observación neurocientífica sería claramente un colonialismo científico. La observación sola no implicaría un colonialismo científico, sino más bien lo que ésta conlleva: la traducción de esas imágenes en datos, y su consecuente cálculo, medición, control y manipulación.

\subsection{Las Inteligencias Múltiples: ¿un falso neuromito?}

En el apartado anterior se ha realizado una breve presentación de los neuromitos y se ha incidido en la cuestión sobre . el carácter de las evidencias neurocientíficas, su poder de seducción y el riesgo que implica deshumanizar la educación a través de un colonialismo científico. A continuación, se tratará el debate sobre la Teoría de las Inteligencias Múltiples (TIE en adelante) de H. Gardner, incidiendo en el debate sobre su consideración o no como neuromito, así como las justificaciones pedagógicas que darían utilidad a esta teoría en la práctica educativa.

La evidencia empírica neurocientífica, así como la justificación educativa que darían soporte a la teoría de las TIE de H. Gardner (1993, 1994) suponen una fuente de controversias. Para algunos, se trataría de un neuromito (Dekker et al., 2012, p. 2; Tokuhama-Espinosa, 2011; Geake, 2008, p. 126; Waterhouse, 2006), mientras que para otros, no lo sería por las evidencias neurocientíficas que la apoyan (Sylwester, 2006; Shearer, 2017, 2020) . Para otros, la utilidad que tiene dentro del proceso de enseñanza-aprendizaje prevalecería por encima de que fuera considerada o no un neuromito (Carillo \& López, 2014; Ferrándiz, et al., 2008; Ferrándiz, et al., 2006; Flórez, 2016; Guzman \& Castro, 2005; Luca, 2004; Shearer, 2018). De hecho, el informe de 
la OECD no lo nombra como un neuromito, en lo que supuso una de las primeras revisiones sistemáticas de éstos en el campo de la neuroeducación (OECD, 2002).

Desde el punto de vista conceptual, el término inteligencia remite a una de las dificultades comentadas anteriormente de la neuroeducación, pues no habría un acuerdo compartido sobre el mismo concepto en la neurociencia y en la educación. El objetivo de este artículo no es hacer una revisión exhaustiva del término, pero sí mostrar cómo esta pugna — entre ser considerado o no un neuromito-, puede ejemplificar el proceder de la neuroeducación. Es decir, ¿quién tiene finalmente la última palabra? ¿Qué prevalecería en este caso, la utilidad y soporte que aporta a la educación o la correspondiente evidencia que lo justifique a nivel cerebral?

El objetivo de las TIE de Gardner fue mostrar su oposición a una concepción unitaria de inteligencia general. Su obra se presenta como una revisión de la inteligencia, desde una perspectiva realmente interdisciplinar. En contra de los tratamientos que hasta los años 90 del siglo XX se había dado a la inteligencia, especialmente en psicometría — como si fuera algo medible y comparable como el CI-, Gardner no solo intentó definirla sino ampliar y reformular la idea de intelecto humano, para poder diseñar formas más apropiadas de evaluarla y educar conforme a ella (Gardner, 1994, p. 36). Es decir, la justificación que da principalmente a su obra es de carácter educativo. Aunque es importante destacar que esta teoría no es nueva, y se podría encontrar en L. L. Thurstone, uno de los primeros en teorizar sobre diferentes tipos de inteligencia — teoría de los vectores de la mente-, usando un método factorial mediante el cual identificó siete tipos: espacial, numérica, fluidez verbal, comprensión verbal, velocidad perceptiva, razonamiento abstracto y memoria asociativa.

No obstante, también alude a evidencias neurológicas (Gardner \& Moran, 2006). En opinión de P. Howard-Jones, estas evidencias no serían suficientes para justificar esta propuesta como una teoría porque el procesamiento general del cerebro es demasiado complejo y no se pueden aislar capacidades concretas a partir de vías concretas de procesamiento cerebral:

La neurociencia cognitiva estudia el cerebro en términos de procesos (visión, audición, olfato, etc.), pero no en los de inteligencia visual, inteligencia auditiva o inteligencia olfativa. En el ámbito de la neurociencia, no parece exacto ni útil reducir la extensa gama de diferencias individuales complejas, a niveles neural y cognitivo, a un número limitado de capacidades (Howard-Jones, 2011, p. 56).

Contrariamente a las palabras de Howard-Jones, la inteligencia para H. Gardner es: "la capacidad de resolver problemas, o de crear, productos, que sean valiosos en uno o más ambientes culturales» (Gardner, 1994, p. 10). Se trata de una definición que nada dice acerca de las fuentes de tales capacidades o de los medios adecuados para medirlas. Hay que tener en cuenta además, que el propio autor advierte que tanto su definición como la lista que da de inteligencias, no es absoluta ni irrefutable, y que pueden existir reajustes, pues «jamás la ciencia da una respuesta completamente 
correcta y definitiva» (Gardner, 1994, p. 95), pero que su teoría intenta responder a una mejor clasificación de las competencias intelectuales humanas, porque el estudio de la educación necesita revisarse y organizarse. Yendo aún más allá, hacia la propia génesis del término "inteligencia», el autor cree que la hemos empleado tan a menudo y la hemos intentado hacer tan mesurable, que la entendemos como algo tangible. Parece ser que nos hemos adherido al concepto de inteligencia porque nos ayuda a hacer frente a diversas situaciones (Gardner, 1994, p. 105).

Las inteligencias no deben ser consideradas, según Gardner, en términos medibles o evaluadores. Más bien, en vez de hacer de la inteligencia algo tangible y medible — desde fuera hacia dentro del ser humano-, deberíamos pensar el término desde dentro del ser humano hacia fuera. Es decir, preguntarnos por qué es necesario hablar de inteligencia. Lo es precisamente por la inmanente sensibilidad del ser humano a un determinado contenido información del medio que le rodea.

Cuando se presenta determinado modo de información, se ponen en actividad diversos mecanismos en el sistema nervioso para realizar operaciones específicas sobre ella. Y el uso repetido de la elaboración e interacción entre estos diversos dispositivos de cómputo, a su debido tiempo fluyen formas de conocimiento que estaríamos dispuestos a denominar «inteligentes» (Gardner, 1994, p. 329).

Cuando habla de los diversos mecanismos que se activan, no se está refiriendo a los sentidos, pues las inteligencias no son equivalentes a los sistemas sensoriales, más bien se realizan por más de un sistema sensorial (Gardner, 1994, p. 103), al contrario de lo que Howard-Jones supone. Además, este autor parece caer en la confusión entre lo conceptual y lo determinado. Esta confusión conlleva a incurrir en la conocida como falacia mereológica y que consiste en atribuir la capacidad o función a una parte que solo se puede atribuir de forma correcta al conjunto de la que ésta forma parte (Bennet \& Hacker, 2003). En efecto, Gardner se refiere a las inteligencias como manifestaciones de los subprocesos neurológicos, pero no a los subprocesos en sí: «las inteligencias deben ser consideradas como entidades en un determinado nivel de generalidad, más amplio que los mecanismos de computación sumamente específicos (...), aunque más estrechos que las capacidades generales, como el análisis, síntesis, o un sentido del yo» (Gardner, 1994, p. 103).

Dejando de lado las evidencias neurocientíficas, parece que ni siquiera las razones educativas y las posibilidades que ofrece la teoría serían suficientes para otros autores, como en el caso de J. Geake, quien realiza una doble crítica contra las IM. En primer lugar se refiere a ellas como algo poco novedoso. Para ello, se remonta a la paideia platónica y compara las TIE con las ciencias propedéuticas que debía estudiar el filósofo-gobernante: lógica, retórica, aritmética, geometría, astronomía, música y danza (Jaeger, 1990), y las asemeja a las inteligencias de H. Gardner: lógica-matemática, verbal, interpersonal, espacial, musical, de movimiento 
corporal, e intrapersonal ${ }^{1}$. Básicamente para Geake, Gardner «recicla a Platón» (Geake, 2008, p. 126). Sin embargo, en esta acusación no es del todo justa.

En primer lugar, H. Gardner no habla de una inteligencia verbal, sino lingüística (Gardner, 1994, p. 109-136), ya que también contempla el lenguaje escrito. También en este sentido, no habla únicamente de una inteligencia de movimiento corporal, sino más concretamente de una inteligencia cinestésicocorporal, porque no solo contempla el movimiento del cuerpo, sino también la expresión del mismo, que iría más allá de lo meramente funcional (Gardner, 1994, p. 252-285).

En segundo lugar, Geake confunde las TIE con las asignaturas o materias curriculares. En este sentido, Gardner ya recogió varias referencias a la filosofía platónica como interés por las variedades de conocimiento racional y formas de conocimiento (Gardner, 1994, p. 37, 46), y advirtió también de los peligros de dicha confusión:

En lo individual, es conveniente hablar de una o más inteligencias humanas, o de tendencias intelectuales del hombre, que forman parte de nuestras facultades. Estas inteligencias se pueden concebir en términos neurobiológicos. Los seres humanos nacen en culturas que agrupan una enorme cantidad de ámbitos: disciplinas, ocupaciones y otras empresas que podemos aprender y sobre las que podemos ser evaluados según el nivel de destreza que hayamos alcanzado (Gardner, 1994, pp. 15-16).

Teniendo en cuenta estas palabras, las inteligencias no son lo mismo que los ámbitos o campos, cosa que Geake parece confundir. Para Gardner, las inteligencias son competencias humanas relativamente autónomas, que si bien pueden dar lugar a que se diseñe un currículo como la paideia platónica u otros, no tienen por qué ser lo mismo forzosamente. De hecho, en caso de relacionarse con un concepto pedagógico, no serían los contenidos o materias curriculares, sino más bien las competencias.

Más arriba ya se ha resaltado que, tanto la actual ley como la propuesta de la nueva ley de educación, tienen un claro enfoque hacia las competencias en lugar de hacia los contenidos. Las competencias clave para el aprendizaje permanente se regulan según la Recomendación 2006/962/CE del Parlamento y Europeo y del Consejo, de 18 de diciembre de 2006 (Diario oficial de la Unión Europea, 2006), y de acuerdo a las disposiciones de la Orden ECD/65/2015, de 21 de enero, por la que se describen las relaciones entre las competencias, los contenidos y los criterios de evaluación de la educación primaria, la educación secundaria obligatoria y el bachillerato (Ministerio de Educación, Cultura y Deporte, 2015). Ambos niveles legales - europeo y nacional - insisten en la necesidad de que la ciudadanía adquiera las competencias básicas como una condición indispensable para alcanzar un pleno

1. En una reformulación posterior a la original de 1983, Gardner ha ampliado el número de inteligencias, haciendo referencia a la naturalista y la existencial (Gardner, 1999). 
desarrollo personal, social y profesional que se ajuste a las demandas de mundo globalizado y haga posible el desarrollo económico vinculado al conocimiento.

En este sentido, teniendo en cuenta estas palabras, las TIE de H. Gardner podrían proporcionar una buena base teórica para la justificación de esta ley educativa y ampliar el enfoque de las competencias. Se podrían dar tres razones para ello. La primera es que las IM contemplan el término competencia en un sentido mucho más amplio, no solo para la resolución de problemas sino también como diseño, creación, correlación, y en definitivo núcleo central del intelecto humano (Gardner, 1994, pp. 36-37). La segunda, porque las IM se correlacionan con las competencias diseñadas en el currículo. No se reducen a ellas, sino que las complementan y las completan en su dimensión humana.

TABLA 1

Relación entre las competencias curriculares y las Inteligencias Múltiples

\begin{tabular}{|l|l|}
\hline \multicolumn{1}{|c|}{ Competencias curriculares } & \multicolumn{1}{c|}{ Inteligencias Múltiples } \\
\hline $\begin{array}{l}\text { Competencia en comunicación lingüística } \\
\text { básicas en ciencia y tecnología }\end{array}$ & Inteligencia lingüística \\
\hline $\begin{array}{l}\text { Tratamiento de la información y la } \\
\text { competencia digital }\end{array}$ & Inteligencia lógico-matemática \\
\cline { 1 - 1 } $\begin{array}{l}\text { Competencia en el conocimiento y la } \\
\text { interacción con el mundo físico }\end{array}$ & $\begin{array}{l}\text { Inteligencia espacial } \\
\text { Inteligencia naturalista }\end{array}$ \\
\hline Competencia social y cívica & \\
\cline { 1 - 1 } Autonomía e iniciativa personal & Inteligencia personal \\
\cline { 1 - 2 } Competencia para aprender a aprender & Inteligencia existencial \\
\cline { 1 - 2 } $\begin{array}{l}\text { Sentido de iniciativa y espíritu } \\
\text { emprendedor }\end{array}$ & Inteligencia música y cinestésicocorporal \\
\hline Conciencia y expresiones culturales &
\end{tabular}

Fuente: Elaboración propia a partir de

Ministerio de Educación Cultura y Deporte (2015) y Gardner (1994)

La tercera, porque las TIE se complementan mejor con una concepción de la evaluación que supere la evaluación únicamente sumativa de conocimientos y se transforme en una evaluación transformadora de procesos desde los estándares de aprendizaje (Monereo, 2009; Sans Martín, 2008; Gibbs \& Simpson, 2009). Según Gardner: "Solo si ampliamos y reformulamos nuestra idea de lo que cuenta como intelecto humano podremos diseñar formas más apropiadas de evaluar o educarla la inteligencia» (Gardner, 1994, p. 36). 


\section{ConClusiones}

El cambio general de dirección en el proceso de enseñanza-aprendizaje ha posibilitado la entrada de las neurociencias en la educación. La orientación hacia las competencias y modelos metodológicos más holísticos y menos heurísticos y enfocados hacia contenidos ha posibilitado, en parte, un diálogo interdisciplinar con las neurociencias, entre otras disciplinas. La neuroeducación se ha ido desarrollando como una ciencia interdisciplinar, realizando aportaciones muy interesantes para el proceder educativo, especialmente las de su valor descriptivo del cerebro o en el aspecto de detección de trastornos durante el proceso de aprendizaje. Como una de las principales aportaciones destacan los neuromitos, o creencias generalizadas que carecen de base empírica neurocientífica. A pesar de la gran utilidad de las neurociencias, es necesaria una aceptación crítica de sus descubrimientos, incluidos los motivos por los cuales aceptamos la catalogación de una teoría como neuromito. Esta aceptación crítica implica no solamente preguntarse por la evidencia neurocientífica en sí, sino en la utilidad humana de las teorías o metodologías que puedan ser identificadas como neuromitos. De lo contrario, se podría caer en un colonialismo científico desde las neurociencias hacia la educación.

El ejemplo sobre el debate alrededor de las teorías múltiples de Gardner ejemplifica varios puntos importantes de esta relación, y contribuyen al objetivo principal del texto que, cabe recordar, es la reflexión crítica sobre la relación entre la neurociencia y la educación, especialmente en la aceptación o no de los neuromitos. En primer lugar, las evaluaciones de las pruebas científicas que refutan la teoría de las TIE continúan siendo controvertidas. Por lo que la supuesta objetividad neurocientífica está sujeta también a la interpretación humana. En segundo lugar, dicha interpretación no puede dejar de lado que defender lo más útil para el ser humano no es siempre una cuestión técnica, sino humanística. Es decir, sería un contrasentido que se quiera apostar por una educación cada vez más comprometida con los valores humanos, la conciencia crítica y la formación ética y cívica, y que las decisiones sobre las metodologías se tomen unilateralmente dentro de una ciencia supuestamente interdisciplinar como es la neuroeducación. El proceso de enseñanza-aprendizaje excede ampliamente los límites de un reduccionismo neurocientífico que tome solo por correcto la evidencia de lo que esté bajo la lente de la experimentación científica.

\section{REFERENCIAS BIBLIOGRÁFICAS}

Adolphs, R. (2010). Conceptual challenges and Directions for Social Neurosciences. Neuron, 65, 752-767. https://doi.org/10.1016/j.neuron.2010.03.006

Ansari, D. (2005). Time to use neuroscience findings in teacher training. Nature, 437, 26. https://doi.org/10.1038/437026a 
Ansari, D., \& Coch, D. (2006). Bridges over troubled waters: education and cognitive neuroscience. Trends in Cognitive Sciences, 10, 146-151. https://doi.org/10.1016/j.tics2066.02.007

Ansari, D., Coch, D., \& De Smedt, B. (2011). Connecting Education and Cognitive Neuroscience: Where will the journey take us? Educational Philosophy and Theory, 43(1), 37-42. https://doi.org/10.1111/j.1469-5812.2010.00705.x

Ansari, D., De Smedt, B., \& Grabner, R. (2012). Neuroeducation: a critical overview of an emerging field. Neuroethics, 5(2), 105-117. https://doi.org/10.1007/s12152-011-9119-3

Battro, A. M. \& Cardinali, D. P. (1996). Más cerebro en la educación. La Nación.

Bauman, Z. (1993). Postmodern ethics. Blackwell.

Bennet, M., \& Hacker, P. (2003). Philosophical Foundations of Neuroscience. Blackwell.

Boletín Oficial del Estado (2020). Ley Orgánica 3/2020, de 29 de diciembre, por la que se modifica La Ley Orgánica 2/2006, de 3 de mayo, de Educación. https://www.boe.es/ eli/es/lo/2020/12/29/3

Bowers, J. S. (2016). The practical and principled problems with educational neuroscience. Psychological Review, 123(5), 600-612. https://doi.org/10.1037/rev0000025

Bruer, J. T. (1997). Education and the Brain: A Bridge Too Far. Educational Researcher, 26(8), 4-16. https://doi.org/10.3102/0013189X026008004

Bruer, J. T. (2005). Building bridges in neuroeducation. En A. M. Battro, K. W. Fischer, \& P. J. Léna (Eds.), The educated brain. Essays in neuroeducation (pp. 43-58). Cambridge University Press.

Campbell, S. R. (2011). Educational Neuroscience: Motivations, methodology and implications. Educational Philosophy and Theory, 43(1), 7-16. https://doi.org/10.1111/j.14695812.2010.00701.x

Campos, A. L. (2010). Neuroeducación: uniendo las neurociencias y la educación en la búsqueda del desarrollo humano.La educ@ción. Revista Digital, 143. http://www. educoea.org/portal/la_educacion_digital/laeducacion_143/articles/neuroeducacion.pdf

Carrillo, M. E., y López, A. (2014). La teoría de las inteligencias múltiples en la enseñanza de las lenguas. Contextos Educativos. Revista de Educación, 17, 79-89.

Cortina, A. (2011). Neuroética y neuropolítica. Sugerencias para la educación moral. Tecnos.

Darder, P., \& Bach, E. (2006). Aportaciones para repensar la teoría y la práctica educativas desde las emociones. Teoría de la Educación. Revista Interuniversitaria, 18, 55-84. https://doi.org/10.14201/3202

Davis, A. (2004). The credentials of brain-based learning. Journal of Philosophy of Education, 38, 21-36. https://doi.org/10.1111/j.0309-8249.2004.00361.x

Dekker, S., Lee, N., Howard-Jones, P., \& Jolles, J. (2012). Neuromyths in education: Prevalence and predictors of misconceptions among teachers. Frontiers in Psychology, 3, 1-8. https:// doi.org/10.3389/fpsyg.2012.00429

Dewey, J. (1995). Democracia y educación: una introducción a la filosofía de la educación. Morata.

Diario Oficial de la Unión Europea. (2006). Recomendación del Parlamento Europeo y del Consejo, de 18 de diciembre de 2006, sobre las competencias clave para el aprendizaje permanente. 2006/962/CE. https://www.boe.es/doue/2006/394/L00010-00018.pdf 
Dündar, S., \& Gündüz, N. (2016). Misconceptions regarding the brain: the neuromyths of preservice teachers. Mind, Brain, and Education 10(4), 212-232. https://doi.org/10.1111/ mbe.12119

Escudero, J. M. (2009). Las caras ocultas del fracaso escolar. Organización y gestión educativa: Revista del Fórum Europeo de Administradores de la Educación.17(5), 19-23.

Ferrándiz, C., Bermejo, R., Sainz, M., Ferrando, M., y Prieto, M. D. (2008). Estudio del razonamiento lógico-matemático desde el modelo de las inteligencias múltiples. Anales De Psicología, 24(2), 213-222. https://www.um.es/analesps/v24/v24_2/05-24_2.pdf

Ferrándiz, C., Prieto, M. D., Bermejo, M. R., y Ferrando, M. (2006). Fundamentos psicopedagógicos de las inteligencias múltiples. Revista española de pedagogía, 64(233), 5-19. https://revistadepedagogia.org/wp-content/uploads/2007/05/FundamentosPsicopedagogicosDeLasInteligencias.pdf

Ferrero, M., Garaizar, P., \& Vadillo, M. A. (2016). Neuromyths in Education: Prevalence among Spanish teachers and an exploration of cross-cultural variation. Frontiers in Human Neuroscience. https://doi.org/10.3389/fnhum.2016.00496

Flórez, J. (2016). Neurodiversidad, discapacidad e inteligencias múltiples. Revista Síndrome de Down, 33, 59-64. http://revistadown.downcantabria.com/2016/06/01/neurodiversidaddiscapacidad-e-inteligencias-multiples/

Formosinho, M., Reis, C., \& De Jesús, P. R. (2013). Educational sciences: towards a theoretical rebirth beyond reductionisms. Teoría de la Educación. Revista Interuniversitaria, 25(1), 47-62. https://doi.org/10.14201/11149

Fuentes, A., y Risso, A. (2015). Evaluación de conocimientos y actitudes sobre neuromitos en futuros/as maestros/as. Revista de Estudios e Investigación en Psicología y Educación, 6(06), 193. https://doi.org/10.17979/reipe.2015.0.06.530

García-Marzá, D. (2012). Neuropolítica. Una mirada crítica sobre el neuropoder. En A. Cortina (Ed.), Neurofilosofía práctica (pp. 77-97). Comares.

García-Marzá, D. (2016). Neuroética aplicada: las consecuencias prácticas del neuropositivismo. Pensamiento. Revista de Información e Investigación Filosófica, 72(273), 881-900. https://doi.org/10.14422/pen.v72.i273.y2016.007

Gardner, H., \& Moran, S. (2006). The science of multiple intelligences theory: A response to Lynn Waterhouse. Educational Psychologist, 41(4), 227-232. https://doi.org/10.1207/ s15326985ep4104_2

Gardner, H. (1993). Multiple Intelligences: The theory in practice. Basic Books.

Gardner, H. (1994). Estructura de la mente. La teoría de las inteligencias múltiples. FCE.

Gardner, H. (1999). Intelligence Reframed. Basic Books.

Geake, J. G. (2008). Neuromythologies in education. Educational Research, 50(2), 123-133. https://doi.org/10.1080/00131880802082518

Gibbs, G., \& Simpson, C. (2009). Condiciones para una evaluación continua favorecedora del aprendizaje. Octaedro.

Gleichgerrcht, E., Lira Luttges, B., Salvarezza, F., \& Campos, A. L. (2015). Educational Neuromyths among teachers in Latin America. Mind, Brain, and Education, 9(3), 170-178. https://doi.org/10.1111/mbe.12086 
Goswami, U. (2006). Neuroscience and education: from research to practice? Nature Reviews Neuroscience. 7(5), 406-413. https://doi.org/10.1038/nrn1907

Goswami, U. (2010). Neuroscience and education. British Journal of Educational Psychology, 74, 1-14. https://doi.org/10.1348/000709904322848798

Gracia, J. (2018). El desafío ético de la educación. Dyckinson.

Gruber, D., \& Dickerson, J. A. (2012). Persuasive images in popular science: Testing judgments of scientific reasoning and credibility. Public Understanding of Science. https:// doi.org/10.1177/0963662512454072

Guzmán, B., y Castro, S. (2005). Las inteligencias múltiples en el aula de clases. Revista de Investigación, 58, 177-202. https://dialnet.unirioja.es/servlet/articulo?codigo=2051112

Habermas, J. (2006). Entre naturalismo y religión. Paidós.

Herculano-Houzel, S. (2002). Do you know your brain? A survey on public neuroscience literacy at the closing of the decade of the brain. Neuroscientist, 8(2), 98-110. https:// doi.org/10.1177/107385840200800206

Hermida, M. J., Segretin, M. S., Soni García, A., \& Lipina, S. J. (2016). Conceptions and misconceptions about neuroscience in preschool teachers: a study from Argentina. Educational Research, 58(4), 457-472. https://doi.org/10.1080/00131881.2016.1238585

Howard-Jones, P. (2011). Investigación neuroeducativa. Neurociencia, educación y cerebro: de los contextos a la práctica. La Muralla.

Howard-Jones, P. (2014). Neuroscience and education: myths and messages. Nature Review Neuroscience 15, 817-824. https://doi.org/10.1038/nrn3817

Illes, J., Moser, M. A., McCormick, J., Racine, E. (et al.) (2010). NeuroTalk: Improving the Communication of Neuroscience. Nature Review of Neuroscience, 11, 61-69. https://doi. org/10.1038/nrn2773

Jaeger, W. (1990). Paideia: los ideales de la cultura griega. FCE.

Kandel, E., Markman, H., Matthews, P. M., Yuste, R., \& Koch, C. (2013). Neuroscience thinks big (and collaboratively). Nature Reviews Neuroscience, 14, 659-664. https://doi. org/10.1038/nrn3578

Kim, M., \& Sankey, D. (2017). Philosophy, neuroscience and pre-service teachers' beliefs in neuromyths: A call for remedial action. Educational Philosophy and Theory, 50(13), 1214-1227. https://doi.org/10.1080/00131857.2017.1395736

Koizumi, H. (2008). Developing the Brain: A functional-imaging based approach to learning and educational sciences. En A. M. Battro, K. W. Fischer, \& P. J. Léna (Eds.), The educated brain. Essays in neuroeducation (pp. 166-180). Cambridge University Press.

Luca, S. L. (2004). El docente y las inteligencias múltiples. Revista Iberoamericana De Educación, 34(1), 1-12. https://doi.org/10.35362/rie3412884

Marina, J. A. (2012). El diálogo entre Neurociencia y Educación. Participación Educativa. Número extraordinario: La investigación sobre el cerebro y la mejora de la educación, 1, 7-13. http://ntic.educacion.es/cee/revista/pdfs/RPE_DIC2012_02Marina.pdf

McCabe, D. P., \& Castel, A. D. (2008). Seeing is believing: The effect of brain images on judgments of scientific reasoning. Cognition, 107, 343-352. https://doi.org/10.1016/j. cognition.2007.07.017 
Medel Montero, M., y Camacho Conde, J. A. (2019). La neurociencia aplicada en el ámbito educativo. El estudio de los neuromitos. International Journal of New Education, 2(1). https://doi.org/10.24310/IJNE2.1.2019.6559

Ministerio de Educación, Cultura y Deporte (2015). Orden ECD/65/2015, de 21 de enero, por la que se describen las relaciones entre las competencias, los contenidos y los criterios de evaluación de la educación primaria, la educación secundaria obligatoria y el bachillerato. BOE-A-2015-738. https://boe.es/buscar/pdf/2015/BOE-A-2015-738-consolidado.pdf

Monereo, C. (2009). La autenticidad de la evaluación. En M. Castelló (Coord.), La evaluación auténtica en la enseñanza secundaria y universitaria: investigación e innovación (pp.15-28). Edebé.

Organization for Economic Co-operation and Development [OECD] (2002). Understanding the Brain. The birth of a learning science. OECD Publishing. https://www.oecd.org/ site/educeri21st/40554190.pdf

Pallarés-Domínguez, D. (2015). Hacia una conceptualización dialógica de la neuroeducación. Participación Educativa, 4(7), 133-141. https://sede.educacion.gob.es/publiventa/ descarga.action?f_codigo_agc $=17395$

Pallarés-Domínguez, D. (2016). Neuroeducación en diálogo: neuromitos en el proceso de enseñanza-aprendizaje y en la educación moral. Pensamiento. Revista de Investigación e Información Filosófica, 72(273), 941-958 https://doi.org/10.14422/pen.v72.i273.y2016.010

Pallarés-Domínguez, D. (2019). Moral Neuroeducation: Proactive Epigenesis and Poverty. En P. Calvo \& J. Gracia-Calandín (Eds.), Moral Neuroeducation for a Democratic and Pluralistic Society (pp. 157-173). Springer. https://doi.org/10.1007/978-3-030-22562-9

Pasquinelli, E. (2012). Neuromyths: why do they exist and persist? Mind, Brain, and Education, 6(2), 89-96. https://doi.org/10.1111/j.1751-228x.2012.01141.x

Racine, E., Bar-Ilan, O., \& Illes, J. (2005). fMRI in the public eye. Nature Reviews Neuroscience, 6(2), 159-164. https://doi.org/10.1038/nrn1609

Rogero, J. (2015). Evaluar en la escuela pública liberadora e inclusiva. Rescoldos: Revista de Diálogo Social, 33, 7-18.

Sans Martín, A. (2008). La evaluación de los aprendizajes: la construcción de instrumentos. Octaedro.

Schumacher, R. (2007). The brain is not enough: Potential and limits in integrating neuroscience and pedagogy. Analyse und Kritik, 29, 38-46. https://doi.org/10.1515/auk-2007-0103

Shearer, B. (2017). The neuroscience of intelligence: empirical support for the theory of multiple intelligences? Trends in Neuroscience and Education, 6, 211-223. https://doi. org/10.1016/j.tine.2017.02.002

Shearer, B. (2018). Multiple Intelligences in Teaching and Education: Lessons Learned from Neuroscience. J. Intell, 6(3), 38. https://doi.org/10.3390/jintelligence6030038

Shearer, B. (2020). Multiple Intelligences in Gifted and Talented Education: Lessons Learned From Neuroscience After 35 Years. Roeper Review, 42(1) https://doi.org/10.1080/0278 3193.2019.1690079

Sylwester, R. (2006). Cognitive Neuroscience Discoveries and Educational Practices. School Administrator, 63(11), 32. https://eric.ed.gov/?id=EJ757406 
Tokuhama-Espinosa, T. N. (2011). Mind, Brain and Education Science. A comprehensive guide to the new brain-based teaching. Norton \& Company.

Torrijos-Muelas, M., González-Víllora, S., \& Bodoque-Osma, A. R. (2021). The persistence of the Neuromyths into educational settings: A systematic review. Frontiers Psychology. https://doi.org/10.3389/fpsyg.2020.591923

Waterhouse, L. (2006). Multiple intelligences, the Mozart effect, and emotional intelligence: A critical review. Educational Psychology, 25, 509-516. https://doi.org/10.1207/ s15326985ep4104_1

Weisberg, D. A., Keil, C., Goodstein, J., Rawson, E., \& Gray, J. (2008). The Seductive Allure of Neuroscience Explanations. Journal of Cognitive Neuroscience, 20(3), 470-477. https:// doi.org/10.1162/jocn.2008.20040

Willingham, D. (2009). Three problems in the marriage of neuroscience and education discussion. Cortex, 45(5), 544-545. https://doi.org/10.1016/j.cortex.2008.05.009

Wolfe, P. (2006). Brain-compatible learning: Fad or foundation? School Administrator, 63(11), 10-15.

Zhang, R., Jiang, Y., Dang, B., \& Zhou, A. (2019). Neuromyths in Chinese classrooms: evidence from headmasters in an underdeveloped region of China. Frontiers in Education, 4(8), 1-6. https://doi.org/10.3389/feduc.2019.00008 